\title{
Stabilization of Pan-Tilt Systems Using Acceleration Based LMI-LQR Controller
}

\author{
Sanem Evren Han and Mustafa Unel*
}

\begin{abstract}
This paper extends the previous work on LPV modeling of a pan-tilt system [1] and tackles the robust stabilization problem by employing angular acceleration feedback in an LMI based optimal LQR controller. The state vector of the LPV model is augmented to include the integral of the position errors in addition to joint angles and velocities. Therefore, an extended polytopic quasi-LPV model of the pantilt system is derived. The LMI based optimal LQR controller that utilizes acceleration feedback is synthesized based on the extended LPV model. Since the time varying parameter vector is 4 dimensional, the proposed controller is synthesized by interpolating LMIs at 16 vertices of the polytope. A cascaded nonlinear high gain observer is also designed to estimate reliable positions, velocities and accelerations from noisy encoder measurements. Simulation results show that the proposed LMI based optimal LQR controller outperforms the classical LMI based LQR controller.
\end{abstract}

\section{INTRODUCTION}

High precision stabilization is one of the significant problems in the control of robotic manipulators. It is difficult to apply nonlinear controllers to the robotic manipulators due to the complexity of these controllers. In recent years, linear parameter varying (LPV) models [2] have been widely used as effective tools to control nonlinear multiple input multiple output (MIMO) systems. LPV models are linear systems and depend on time varying measurable scheduling signals.

The advantage of LPV models is to represent many nonlinear systems using linear dynamical relationships and allow the use of well known linear optimal controllers and linear matrix inequalities (LMIs) on these systems. Capturing nonlinear systems using linear relationships leads to development of quasi-LPV model [3]-[4] where the scheduling signals can be the states, inputs or outputs of the system. Polytopic LPV approach [5]-[6] is also a popular technique to synthesize the linear controllers by interpolating LMIs at different vertices of the polytope. LPV models have been used to synthesize various control algorithms such as gain scheduling controllers [7]-[9], $H_{\infty}$ controllers [10]-[11], and LQR controllers [12]-[13].

In this paper, the polytopic quasi-LPV model derived in [1] is extended by including the integral of the position errors in the new state vector. An LMI based optimal LQR controller that utilizes acceleration feedback (AFB) is synthesized based on this LPV model. The acceleration feedback is incorporated into the LMI based LQR controller

*Corresponding Author

Sanem Evren Han and Mustafa Unel are with Faculty of Engineering and Natural Sciences, Sabanci University, Istanbul, Turkey (sanemevren, munel) @sabanciuniv.edu since the effects of external disturbances manifest themselves first in the acceleration signals. Therefore, the proposed controller should provide increased robustness of the system to external disturbances and achieve high precision stabilization. The performance of the proposed controller largely depends on reliable feedback signals. Thus, a cascaded high gain observer (CHGO) is designed to estimate reliable position, velocity and acceleration signals from noisy encoder measurements. Estimated position, velocity and acceleration signals are used in the acceleration based LQR controller. A high fidelity simulation model is constructed by designing realistic encoder and nonlinear pan-tilt models. Simulation results demonstrates the validity and effectiveness of the proposed LMI-LQR controller.

The remainder of this paper is organized as follows: Section II develops a cascaded high gain observer (CHGO) to estimate reliable position, velocity and acceleration signals. In Section III, an extended polytopic quasi-LPV model of a 2 DOF pan-tilt robot is developed and an acceleration based LMI-LQR controller is synthesized based on the proposed LPV model. Section IV provides simulation results where the effectiveness of the proposed control approach is validated on the pan-tilt system. Finally, Section V concludes the paper with important remarks and indicates possible future directions.

\section{ENCODER MODELING AND Estimation By A CASCADED HIGH GAIN OBSERVER}

This section models an encoder and develops the proposed observer to estimate position, velocity and acceleration feedback signals.

\section{A. Encoder Modeling}

Encoders measure joint angles in control applications. Encoders are modeled by corrupting the true sensor measurements with sensor errors as follows:

$$
q_{m}=q_{0}+b_{e}+\mu_{e}
$$

where $q_{m} \in \mathbb{R}^{n}$ is the measured encoder data, $q_{0} \in \mathbb{R}^{n}$ is the true encoder measurement, and $b_{e} \in \mathbb{R}^{n}$ and $\mu_{e} \in \mathbb{R}^{n}$ represent the encoder biases and time correlated noises, respectively.

\section{B. A Cascaded High Gain Observer}

A new high gain observer where two different observers are developed in a cascaded structure is proposed as depicted in Figure 1. This observer provides reliable estimates of 
link positions, $\hat{\zeta}_{o_{1}}$, velocities, $\hat{\sigma}_{o_{1}}$, and accelerations, $\hat{\sigma}_{o_{2}}$, by utilizing noisy position measurements from an encoder.

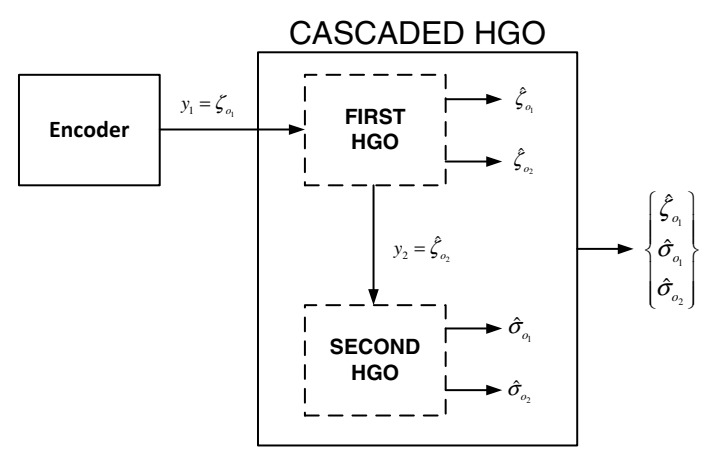

Fig. 1. Block diagram of Cascaded HGO Structure

The first HGO uses position measurements by an encoder to estimate position and velocity signals. The second HGO, on the other hand, utilizes estimated velocities by the first HGO to provide estimates of link accelerations. The dynamics of the first HGO is designed as:

$$
\begin{aligned}
& \dot{\hat{\zeta}}_{o_{1}}=\hat{\zeta}_{o_{2}}+\mathbb{L}_{1}\left(y_{1}-\hat{\zeta}_{o_{1}}\right) \\
& \dot{\zeta}_{o_{2}}=\mathbb{L}_{2}\left(y_{1}-\hat{\zeta}_{o_{1}}\right)
\end{aligned}
$$

where $\hat{\zeta}_{o_{1}} \in \mathbb{R}^{n}$ and $\hat{\zeta}_{o_{2}} \in \mathbb{R}^{n}$ are the estimated link positions and velocities, $\hat{\zeta}_{o}(t)=\left[\begin{array}{ll}\hat{\zeta}_{o_{1}} & \hat{\zeta}_{o_{2}}\end{array}\right]^{T} \in \mathbb{R}^{2 n}$ denotes the observer state vector, $y_{1}=\stackrel{q}{m}_{m} \in \mathbb{R}^{n}$ is the encoder measurement given in (1), and the observer gains should be designed as:

$$
\mathbb{L}_{1}=\frac{\beta_{1}}{\varepsilon_{1}}, \quad \text { and } \quad \mathbb{L}_{2}=\frac{\beta_{2}}{\varepsilon_{1}^{2}}
$$

for some positive constants $\beta_{1}, \beta_{2} \in \mathbb{R}$, and $\varepsilon_{1} \ll 1$. Similarly, the dynamics of the second HGO is as follows:

$$
\begin{aligned}
& \dot{\hat{\sigma}}_{o_{1}}=\hat{\sigma}_{o_{2}}+\mathbb{L}_{3}\left(y_{2}-\hat{\sigma}_{o_{1}}\right) \\
& \dot{\hat{\sigma}}_{o_{2}}=\mathbb{L}_{4}\left(y_{2}-\hat{\sigma}_{o_{1}}\right)
\end{aligned}
$$

where $\hat{\sigma}_{o_{1}} \in \mathbb{R}^{n}$ and $\hat{\sigma}_{o_{2}} \in \mathbb{R}^{n}$ are the estimated link velocities and accelerations, $\hat{\sigma}_{o}(t)=\left[\begin{array}{ll}\hat{\sigma}_{o_{1}} & \hat{\sigma}_{o_{2}}\end{array}\right]^{T} \in \mathbb{R}^{2 n}$ represents the observer state vector, $y_{2}=\hat{\zeta}_{o_{2}} \in \mathbb{R}^{n}$ is the estimated velocity by the first $\mathrm{HGO}$, and $\mathbb{L}_{3}, \mathbb{L}_{4}$ are the observer gains as follows:

$$
\mathbb{L}_{3}=\frac{\beta_{3}}{\varepsilon_{2}}, \quad \text { and } \quad \mathbb{L}_{4}=\frac{\beta_{4}}{\varepsilon_{2}^{2}}
$$

for some positive constants $\beta_{3}, \beta_{4} \in \mathbb{R}$, and $\varepsilon_{2} \ll 1$. These observers are referred as high gain observers because larger observer gains, $\mathbb{L}_{1}, \mathbb{L}_{2}, \mathbb{L}_{3}$, and $\mathbb{L}_{4}$, are used in order to achieve zero estimation errors. High gain observers suffer from a peaking phenomenon due to sufficiently small $\varepsilon_{1}$ and $\varepsilon_{2}$. This phenomenon is handled by saturating the control input. The readers are referred to [14] for the details.

\section{LPV ROBUST CONTROL}

An extended polytopic quasi-LPV model of the pan-tilt system is derived and the proposed LPV model is utilized to synthesize an acceleration based LQR controller.

\section{A. The Extended Polytopic Quasi-LPV Model}

The polytopic quasi-LPV model of the pan-tilt system is derived in [1]. The state vector of this LPV model, $x \in \mathbb{R}^{4}$, is designed as:

$$
x=\left[\begin{array}{llll}
q_{1} & q_{2} & \dot{q}_{1} & \dot{q}_{2}
\end{array}\right]^{T}
$$

where $q$ and $\dot{q}$ are the joint angles and velocities. The readers are referred to [1] for the details about the obtained quasiLPV model. In this work, the state vector is augmented to include the integral of the position errors as follows:

$$
z=\left[\begin{array}{llllll}
q_{1} & q_{2} & \dot{q}_{1} & \dot{q}_{2} & \int\left(r_{1}-q_{1}\right) d t \quad \int\left(r_{2}-q_{2}\right) d t
\end{array}\right]_{(7)}^{T}
$$

with $z \in \mathbb{R}^{6}$ being the extended state vector and the desired joint angles are denoted by $r=\left[\begin{array}{ll}r_{1} & r_{2}\end{array}\right]^{T} \in \mathbb{R}^{2}$. In accordance with the new state vector in (7), an extended polytopic quasi-LPV model is designed as follows:

$$
\begin{gathered}
\dot{z}(t)=E(\phi(t)) z(t)+F(\phi(t)) u(t)+H r(t) \\
y(t)=G(\phi(t)) z(t)+M(\phi(t)) u(t)
\end{gathered}
$$

where

$$
\begin{gathered}
E=\left[\begin{array}{cccccc}
0 & 0 & 1 & 0 & 0 & 0 \\
0 & 0 & 0 & 1 & 0 & 0 \\
0 & 0 & 0 & \phi_{1} & 0 & 0 \\
0 & \phi_{2} & \phi_{3} & 0 & 0 & 0 \\
-1 & 0 & 0 & 0 & 0 & 0 \\
0 & -1 & 0 & 0 & 0 & 0
\end{array}\right], \quad F=\left[\begin{array}{cc}
0 & 0 \\
0 & 0 \\
\phi_{4} & 0 \\
0 & \frac{1}{c} \\
0 & 0 \\
0 & 0
\end{array}\right], \\
H=\left[\begin{array}{lll}
0_{4 \times 2} & I_{2 \times 2}
\end{array}\right]^{T}, \quad G=\left[\begin{array}{ll}
I_{2 \times 2} & 0_{2 \times 4}
\end{array}\right], \quad M=0_{2 \times 2}
\end{gathered}
$$

and $u \in \mathbb{R}^{2}$ defines the control input, $y \in \mathbb{R}^{2}$ is the output. The system matrices $E($.$) and F($.$) depend on the time varying$ parameter vector, $\phi(t) \in \mathbb{R}^{4}$ whose elements are obtained as follows [1]:

$$
\begin{gathered}
\phi_{1}=\frac{\left(b \sin q_{2}+c \sin 2 q_{2}\right) \dot{q}_{1}}{h} \\
\phi_{2}=-\frac{d}{c} \frac{\cos q_{2}}{q_{2}} \\
\phi_{3}=-\frac{1}{2 c}\left(b \sin q_{2}+c \sin 2 q_{2}\right) \dot{q}_{1} \\
\phi_{4}=\frac{1}{h}
\end{gathered}
$$

where $a, b, c$ and $d$ represent dynamic and kinematic parameters of the pan-tilt system.

\section{B. LMI Based LQR Synthesis Based on the Proposed LPV Model}

This section designs an acceleration based LMI-LQR controller on the proposed LPV model. This controller is used to stabilize the nonlinear pan-tilt system and the performance of the developed controller is compared with the classical LMI based LQR controller in Section IV.

The classical LQR controller is designed as

$$
u_{1}=K z
$$


that minimizes the following cost function:

$$
J_{1}=\int\left(z^{T} Q z+u_{1}^{T} R u_{1}\right) d t
$$

where $K \in \mathbb{R}^{2 \times 6}$ is the feedback gain matrix, $Q \in \mathbb{R}^{6 \times 6}$ and $R \in \mathbb{R}^{2 \times 2}$ are the state and control input weighting matrices. These matrices are constant, symmetric and positive-definite, i.e. $Q>0$ and $R>0$. $K$ is obtained by solving the following semidefinite programming problem:

$$
\min \operatorname{tr}(P)
$$

subject to

$$
(E+F K)^{T} P+P(E+F K) \leq-Q-K^{T} R K
$$

where $P \in \mathbb{R}^{6 \times 6}>0$ is the Lyapunov matrix and tr denotes the trace. Non-convex optimization problem in (13)-(14) is converted into a convex problem by multiplying left and right side of (14) with $P^{-1}$ and applying Schur Complement [15]:

$$
\max \operatorname{tr}(Y)
$$

subject to

$$
\begin{aligned}
& {\left[\begin{array}{ccc}
-(E+F K)-(E+F K)^{T} & Y & L^{T} \\
Y & Q^{-1} & 0_{6 \times 2} \\
L & 0_{2 \times 6} & R^{-1}
\end{array}\right] \geq 0} \\
& Y=P^{-1}>0
\end{aligned}
$$

where $L \in \mathbb{R}^{2 \times 6}$ is introduced as $L=K Y$ and $Y \in \mathbb{R}^{6 \times 6}$ is the inverse of the Lyapunov matrix, $Y=P^{-1}$. The feedback matrix can be recovered as:

$$
K=L Y^{-1}
$$

An acceleration based linear state feedback law is designed as

$$
u_{2}=K_{1} z+K_{2} \dot{z}
$$

where $K_{1}, K_{2} \in \mathbb{R}^{2 \times 6}$ are the feedback gain matrices which will be designed by minimizing the following cost function:

$$
J_{2}=\int\left(z^{T} Q_{1} z+\dot{z}^{T} Q_{2} \dot{z}+u_{2}^{T} R u_{2}\right) d t
$$

where the cost function in (12) is modified such that acceleration signals are also included in the new cost function. $Q_{2}>0 \in \mathbb{R}^{6 \times 6}$ is a constant symmetric positive-definite matrix that penalizes the derivative of the state vector. The overall control system is presented in Figure 2. Utilization of both $z$ and $\dot{z}$ leads to redundancy in terms of position and velocity in the controller formulation given by (19). In this work, this redundancy is reduced by selecting smaller weights corresponding to position and velocity terms in $Q_{2}$.

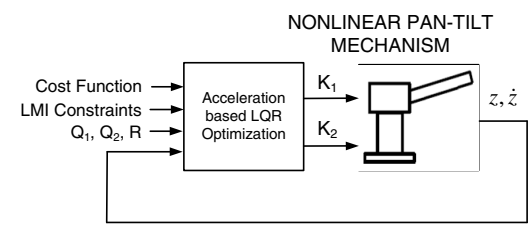

Fig. 2. Control block diagram
The controller in (19) is designed by solving the following semidefinite programming problem:

$$
\min \left(\operatorname{tr}\left(P_{1}\right)+\operatorname{tr}\left(P_{2}\right)\right)
$$

subject to

$$
\begin{aligned}
& \left(E+F K_{1}\right)^{T} P_{1}+P_{1}\left(E+F K_{1}\right) \leq-Q_{1}-K_{1}^{T} R K_{1} \\
& \left(E+F K_{2}\right)^{T} P_{2}+P_{2}\left(E+F K_{2}\right) \leq-Q_{2}-K_{2}^{T} R K_{2}
\end{aligned}
$$

where $P_{1}$, and $P_{2} \in \mathbb{R}^{6 \times 6}>0$ are the Lyapunov matrices, and there exists a first-order, differentiable, positive-definite function $V(t) \in \mathbb{R}$ such that

$$
\begin{aligned}
\dot{V}(t) \leq & -z^{T} Q_{1} z-\dot{z}^{T} Q_{2} \dot{z}-u_{2}^{T} R u_{2}+2 \dot{z}^{T} K_{2}^{T} F P_{1} z \\
& +2 r^{T} H^{T} P_{1} z+2 z^{T} K_{1}^{T} R K_{2} \dot{z} \\
& -\dot{z}^{T}\left[\left(E+F K_{2}\right)^{T} P_{2}+P_{2}\left(E+F K_{2}\right)\right] \dot{z}
\end{aligned}
$$

is satisfied. The following Lyapunov based analysis proves the derivation of the constraints given in (22)-(23) using (24). Proof: A Lyapunov function candidate, $V(t)$ is defined as

$$
V=z^{T} P_{1} z
$$

The following expression is computed by taking the time derivative of (25), and using the assumption given in (24):

$$
\begin{aligned}
& z^{T}\left[\left(E+F K_{1}\right)^{T} P_{1}+P_{1}\left(E+F K_{1}\right)\right] z+\dot{z}^{T}\left[\left(E+F K_{2}\right)^{T} P_{2}\right. \\
& \left.+P_{2}\left(E+F K_{2}\right)\right] \dot{z} \leq-z^{T} Q_{1} z-\dot{z}^{T} Q_{2} \dot{z}-u_{2}^{T} R u_{2} \\
& +2 z^{T} K_{1}^{T} R K_{2} \dot{z}
\end{aligned}
$$

By substituting (19) into (26) and performing cancellations, one obtains that:

$$
\begin{aligned}
& z^{T}\left[\left(E+F K_{1}\right)^{T} P_{1}+P_{1}\left(E+F K_{1}\right)\right] z+\dot{z}^{T}\left[\left(E+F K_{2}\right)^{T} P_{2}\right. \\
& \left.+P_{2}\left(E+F K_{2}\right)\right] \dot{z} \leq-z^{T}\left(Q_{1}+K_{1}^{T} R K_{1}\right) z \\
& -\dot{z}^{T}\left(Q_{2}+K_{2}^{T} R K_{2}\right) \dot{z}
\end{aligned}
$$

The following constraints are derived in order to satisfy the inequality in (26):

$$
\begin{aligned}
& \left(E+F K_{1}\right)^{T} P_{1}+P_{1}\left(E+F K_{1}\right) \leq-Q_{1}-K_{1}^{T} R K_{1} \\
& \left(E+F K_{2}\right)^{T} P_{2}+P_{2}\left(E+F K_{2}\right) \leq-Q_{2}-K_{3}^{T} R K_{2}
\end{aligned}
$$

(21)-(23) is a non-convex optimization problem. It is converted into a convex problem by multiplying left and right side of (22)-(23) with $P_{1}^{-1}$ and $P_{2}^{-1}$, and applying Schur Complement [15]:

$$
\max \left(\operatorname{tr}\left(Y_{1}\right)+\operatorname{tr}\left(Y_{2}\right)\right)
$$

subject to

$$
\begin{gathered}
{\left[\begin{array}{ccc}
-\left(E+F K_{1}\right)-\left(E+F K_{1}\right)^{T} & Y_{1} & L_{1}^{T} \\
Y_{1} & Q_{1}^{-1} & 0_{6 \times 2} \\
L_{1} & 0_{2 \times 6} & R^{-1}
\end{array}\right] \geq 0} \\
{\left[\begin{array}{ccc}
-\left(E+F K_{2}\right)-\left(E+F K_{2}\right)^{T} & Y_{2} & L_{2}^{T} \\
Y_{2} & Q_{2}^{-1} & 0_{6 \times 2} \\
L_{2} & 0_{2 \times 6} & R^{-1}
\end{array}\right] \geq 0}
\end{gathered}
$$


and

$$
Y_{1}=P_{1}^{-1}>0, \quad Y_{2}=P_{2}^{-1}>0
$$

where $L_{1}=K_{1} Y_{1}$ and $L_{2}=K_{2} Y_{2} . Y_{1}$ and $Y_{2}$ are the inverses of the Lyapunov matrices, $Y_{1}=P_{1}^{-1}$ and $Y_{2}=P_{2}^{-1}$. The controller matrices, $K_{1}$ and $K_{2}$ are recovered as

$$
\begin{aligned}
& K_{1}=L_{1} Y_{1}^{-1} \\
& K_{2}=L_{2} Y_{2}^{-1}
\end{aligned}
$$

The robust optimization toolbox YALMIP [16] is used to design the classical LMI based LQR controller in (11) and the proposed controller in (19). These controllers are synthesized based on the developed polytopic quasi-LPV model given in (8)-(9). Since the time varying parameter vector is 4 dimensional in the extended polytopic-quasi LPV model, i.e. $\phi(t) \in \mathbb{R}^{4}$, the total number of vertices is $\lambda=$ $2^{4}=16$. Therefore, the classical LMI based LQR controller is designed by interpolating LMIs given in (16)-(17) at each vertex. Similarly, the proposed controller is developed by interpolating LMIs given in (31)-(33) at each vertex.

\section{Simulation Results}

The physical constraints that are applied to the joints are as follow:

TABLE I

PHYSICAL CONSTRAINTS

\begin{tabular}{ccc} 
Parameter & Minimum Value & Maximum Value \\
\hline$q_{1}$ & $-170^{\circ}$ & $170^{\circ}$ \\
$q_{2}$ & $-85^{\circ}$ & $85^{\circ}$ \\
$\dot{q}_{1}$ & $-150^{\circ} / \mathrm{sec}$ & $150^{\circ} / \mathrm{sec}$ \\
$\dot{q}_{2}$ & $-50^{\circ} / \mathrm{sec}$ & $50^{\circ} / \mathrm{sec}$ \\
\hline
\end{tabular}

Using the physical constraint given in Table I, scheduling position trajectories are designed as quintic polynomials in Figures 3. Since the position trajectories are designed as 5 th degree polynomials, joint velocity trajectories are 4th degree polynomials as shown in Figure 4. The parameter

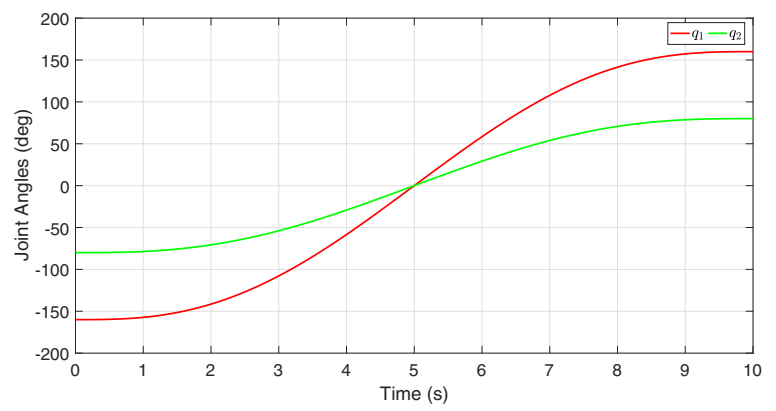

Fig. 3. Scheduling joint position signals

trajectories, $\theta_{j}$, are generated based on (10) in Figures 5-8. $\theta_{1}$ and $\theta_{3}$ depend on $q_{2}$ and $\dot{q}_{1}$. On the other hand, $\theta_{2}$ and $\theta_{4}$ are the function of only $q_{2}$. Due to this dependency, the parameter values have the upper and lower bounds given in Table II.

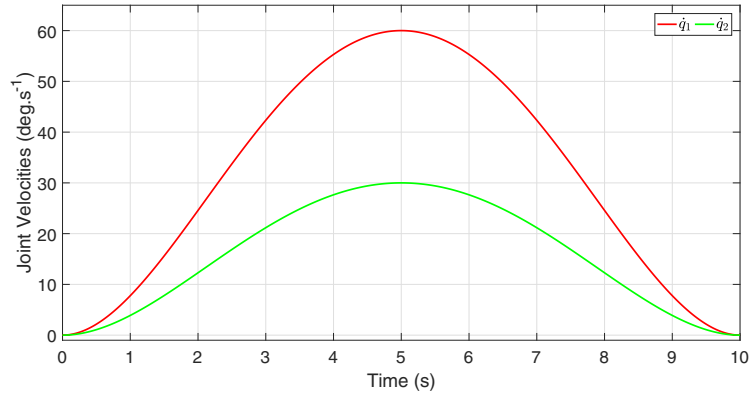

Fig. 4. Scheduling joint velocity signals

TABLE II

UPPER AND LOWER BOUNDS OF THE PARAMETER VECTOR

\begin{tabular}{llcc}
\multicolumn{2}{l}{ Parameter } & Upper Bound & Lower Bound \\
\hline$\theta_{1}$ & $\left(\right.$ rad.sec $\left.^{-1}\right)$ & 0.4225 & -0.4225 \\
$\theta_{2}$ & $(\text { rad.sec })^{-1}$ & -1.2812 & -14.7 \\
$\theta_{3}$ & $($ unitless $)$ & 1.4405 & -1.4405 \\
$\theta_{4}$ & $\left(\text { kg. }^{2}\right)^{-1}$ & 1.2581 & 0.7059 \\
\hline
\end{tabular}

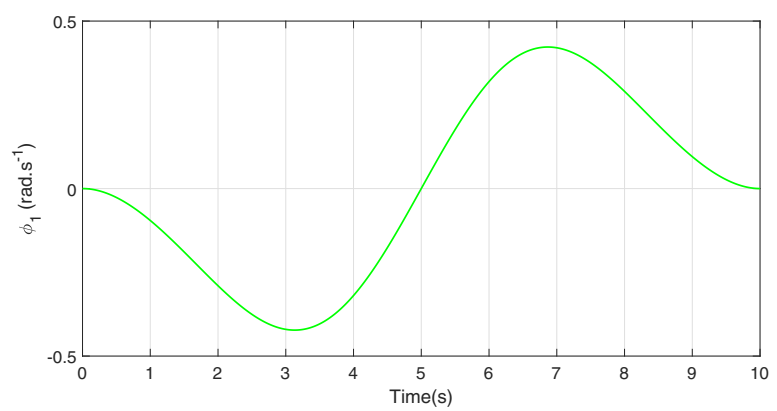

Fig. 5. Parameter trajectory: $\phi_{1}$

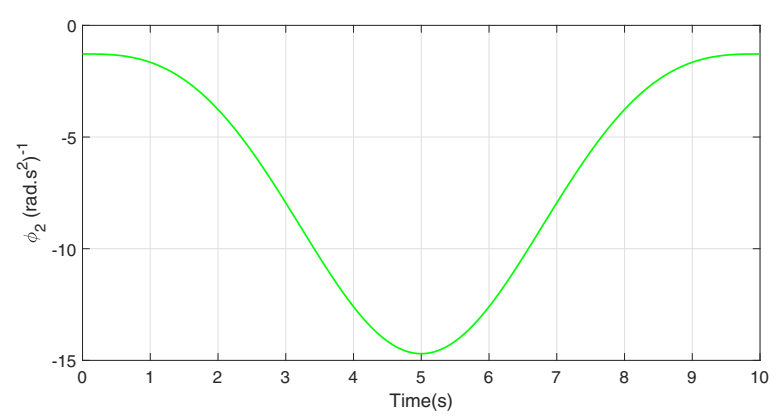

Fig. 6. Parameter trajectory: $\phi_{2}$

External disturbances shown in Figure 9 are applied on the system after the desired positions are reached. These disturbances are modeled as high amplitude step pulses with short durations. The amplitudes of the step pulses are assumed as $10 \mathrm{~N} . \mathrm{m}$ and $15 \mathrm{~N} . \mathrm{m}$. The performance of the proposed controller in (19) is compared with the performance of the classical LQR controller in (11). LQR controller is synthesized based on the developed polytopic quasi-LPV model in (8)-(9). The total number of vertices is $\lambda=2^{4}=16$. The proposed state feedback controller given in (19) is 


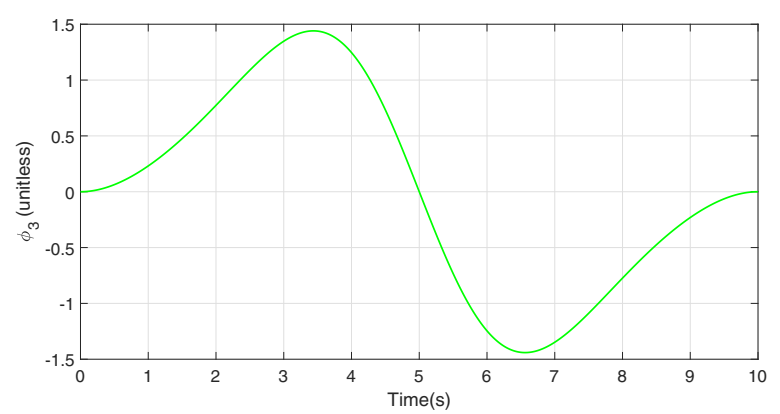

Fig. 7. Parameter trajectory: $\phi_{3}$

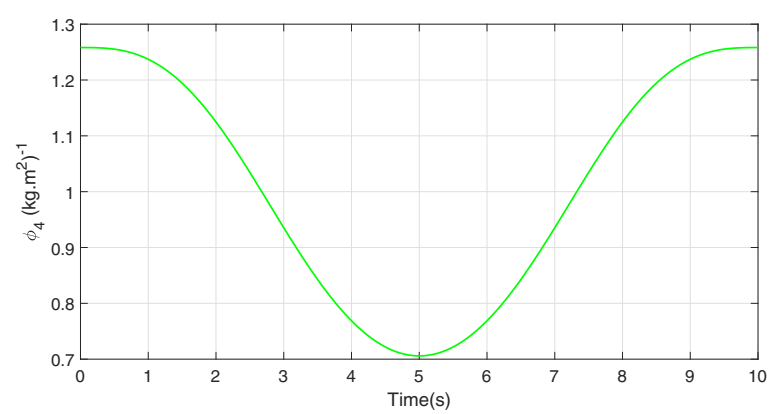

Fig. 8. Parameter trajectory: $\phi_{4}$
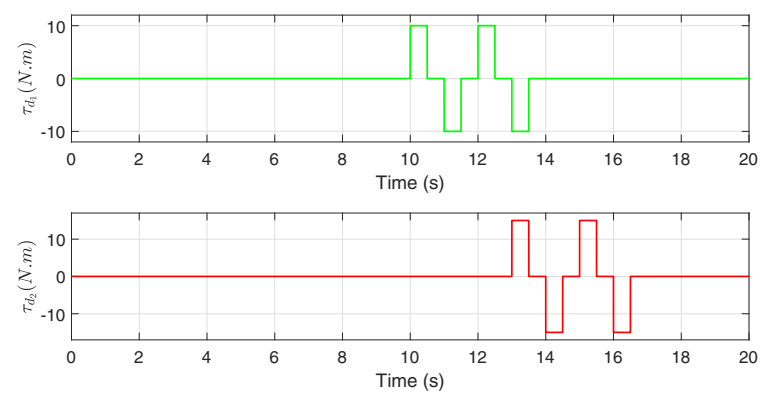

Fig. 9. External disturbances on the pan and tilt axes

designed by interpolating LMIs at each vertex. The elements of the state feedback gain matrix, $K, K_{1}$ and $K_{2}$ are designed based on the weighting matrices, $Q_{1}, Q_{2}$ and $R$ :

$$
Q=Q_{1}=\left[\begin{array}{llllll}
1 & 0 & 0 & 0 & 0 & 0 \\
0 & 1 & 0 & 0 & 0 & 0 \\
0 & 0 & 1 & 0 & 0 & 0 \\
0 & 0 & 0 & 1 & 0 & 0 \\
0 & 0 & 0 & 0 & 5 & 0 \\
0 & 0 & 0 & 0 & 0 & 5
\end{array}\right], \quad R=\left[\begin{array}{cc}
0.001 & 0 \\
0 & 0.01
\end{array}\right]
$$$$
Q_{2}=\left[\begin{array}{cccccc}
0.001 & 0 & 0 & 0 & 0 & 0 \\
0 & 0.001 & 0 & 0 & 0 & 0 \\
0 & 0 & 100 & 0 & 0 & 0 \\
0 & 0 & 0 & 100 & 0 & 0 \\
0 & 0 & 0 & 0 & 0.001 & 0 \\
0 & 0 & 0 & 0 & 0 & 0.001
\end{array}\right]
$$

More weighting is added to the integral of the position errors than the other states in $Q_{1}$ to eliminate the steady state error. $R$ limits the amplitude of the control input and the elements of the matrix $R$ are designed smaller than the elements of $Q_{1}$ matrix. This makes the system sensitive to the states of the system instead of the control input. As it is previously mentioned in Section III, there is a redundancy in the controller due to common position and velocity terms in $z$ and $\dot{z}$. The effect of this redundancy is reduced by choosing the elements of $Q_{2}$ are smaller, $Q_{2_{11}}=Q_{2_{22}}=Q_{2_{55}}=Q_{2_{66}}=$ 0.001 .

Using the system model (8)-(9) and the weighting matrices in (36), the optimal feedback gain matrices, $K, K_{1}$, $K_{2} \in R^{2 \times 6}$, obtained by YALMIP is given in (37)-(39). The performance of the controller is tested on the nonlinear pan-tilt system. Initial joint angles are assumed as 150 deg and $75 \mathrm{deg}$. The desired angles are $r_{1}=65 \mathrm{deg}$ and $r_{2}=15 \mathrm{deg}$. Joint angles converge to reference values as depicted in Figure 10. Joint velocities converge to zero in Figure 11. Higher amplitude oscillations are obtained in all transient responses with the classical controller. As the external disturbances are applied on the system, acceleration feedback (AFB) improves the system responses compared to the case where the acceleration feedback is not used in the controller.
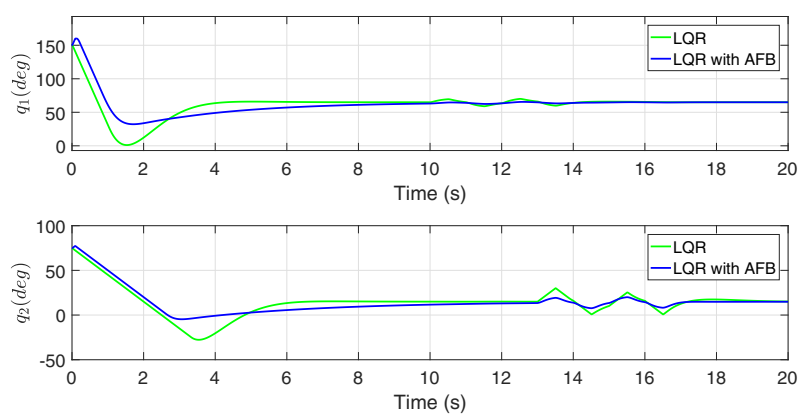

Fig. 10. Output joint angles
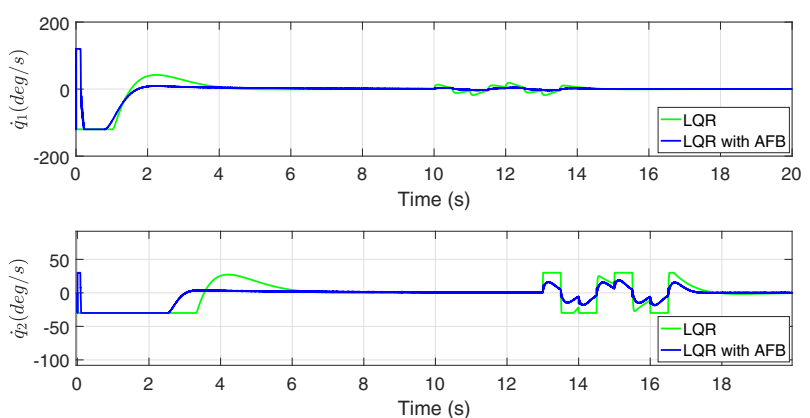

Fig. 11. Output joint velocities

The performance specifications given in Tables III and IV also show that the proposed controller outperforms the classical LMI based LQR controller. 


$$
\begin{aligned}
& K=\left[\begin{array}{cccccc}
-81.54 & -7.83 \times 10^{-11} & -36.22 & -3.6283 \times 10^{-12} & 75.86 & 1.95 \times 10^{-12} \\
-1.05 \times 10^{-11} & -27.71 & -8.85 \times 10^{-12} & -10.61 & 1.73 \times 10^{-11} & 24.70
\end{array}\right] \\
& K_{1}=\left[\begin{array}{cccccc}
-88.31 & -3.43 \times 10^{-11} & -36.51 & -1.26 \times 10^{-12} & 85.42 & -5.67 \times 10^{-11} \\
-3.76 \times 10^{-9} & -30.62 & -3.70 \times 10^{-10} & -10.66 & 2.19 \times 10^{-8} & 29.70
\end{array}\right] \\
& K_{2}=\left[\begin{array}{cccccc}
-135.86 & -2.73 \times 10^{-10} & -23.98 & -2.2578 \times 10^{-11} & 384.34 & 5.86 \times 10^{-9} \\
-3.76 \times 10^{-9} & -24.33 & -3.70 \times 10^{-10} & -3.04 & 2.19 \times 10^{-8} & 105.65
\end{array}\right]
\end{aligned}
$$

TABLE III

Link 1 Performance Specification

\begin{tabular}{ccc}
\hline Transient Responses $(t=1-10 \mathrm{sec})$ & & \\
\hline $\begin{array}{c}\text { Performance } \\
\text { Criteria }\end{array}$ & $\begin{array}{c}\text { Proposed } \\
\text { Controller }\end{array}$ & $\begin{array}{c}\text { Classical } \\
\text { Controller }\end{array}$ \\
\hline Worst Case Position Error (deg) & 20.55 & 55.08 \\
RMS Position Error (deg) & 19.30 & 20.38 \\
RMS Control Input (N.m) & 5.35 & 3.62 \\
\hline System Responses During Disturbances & & \\
\hline Performance & Proposed & Classical \\
Criteria & Controller & Controller \\
\hline Worst Case Position Error (deg) & 1.28 & 4.92 \\
RMS Position Error (deg) & 2.64 & 3.27 \\
RMS Control Input (N.m) & 7.14 & 7.35 \\
\hline
\end{tabular}

TABLE IV

Link 2 PERformanCE SPECIFICATION

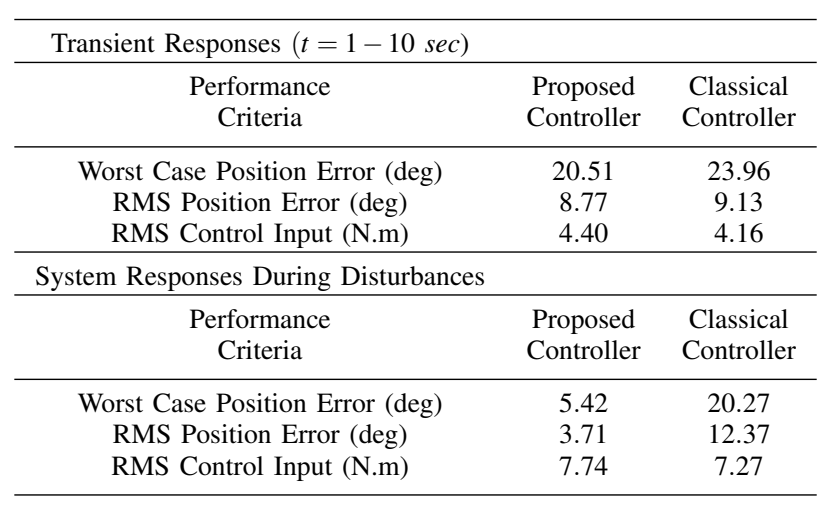

\section{CONCLUSiOnS AND Future Works}

An extended polytopic quasi-LPV model of the nonlinear pan-tilt system has been derived to synthesize acceleration based LQR controller for the pan-tilt stabilization. Acceleration signals are estimated by a new high gain observer structure where two different observers are developed in a cascaded structure. The first $\mathrm{HGO}$ uses position measurements by an encoder to estimate reliable position and velocity information. The second HGO, on the other hand, utilizes estimated velocities by the first HGO to provide estimates of acceleration signals. Obtained estimates by the proposed HGO structure are used in the proposed controller. The feedback gain matrix is designed by interpolating LMIs at each vertex of the polytopic model. Pulse disturbances are exerted on the system to compare the performance of the proposed LQR controller with the classical one. Smaller amplitude oscillations are observed as acceleration feedback is utilized in the optimal controller.

\section{REFERENCES}

[1] S. Evren and M. Unel, "Stabilization of a Pan-Tilt System Using a Polytopic Quasi-LPV Model and LQR Control," IECON, 2016.

[2] J. S. Shamma and M. Athans, "Analysis of gain scheduled control for nonlinear plants," IEEE Transactions on Automatic Control, vol. 35, no. 8, pp. 898-907, 1990.

[3] P. S. G. Cisneros, S. Voss, H. Werner, "Efficient nonlinear model predictive vontrol via quasi-LPV representation," IEEE Conference on Decision and Control, 2016.

[4] D. Rotondo, F. Nejjari, V. Puig, "Quasi-LPV modeling, identification and control of a twin rotor MIMO system," Control Engineering Practice, vol. 21, no. 6, pp. 829-846, 2013.

[5] N. Wang, C. Hu, Q. Xie, "Polytopic LPV-based robust model predictive control with varying horizons for air-breathing hypersonic vehicles," Chinese Control and Decision Conference, 2016.

[6] M. Rodrigues, M. Sahnoun, D. Theilliol, J. C. Ponsart "Sensor fault detection and isolation filter for polytopic LPV systems: A winding machine application," Journal of Process Control, vol. 23, no. 6, pp. 805-816, 2013.

[7] Y. Huang, C. Sun, C. Qian, J. Zhang, L. Wang, "Polytopic LPV modeling and gain-scheduled switching control for a flexible airbreathing hypersonic vehicle," Journal of Systems Engineering and Electronics, vol. 24, no. 1, pp. 118-127, 2013.

[8] P. Ballesteros, X. Shu, C. Bohn, "Active Control of Engine-Induced Vibrations in Automotive Vehicles through LPV Gain Scheduling," International Journal of Passenger Cars-Electronic and Electrical Systems, vol. 7, no. 1, pp. 264-272, 2014.

[9] X. Shu, P. Ballesteros, W. Heins, C. Bohn, "Design of structured discrete-time LPV gain-scheduling controllers through state augmentation and partial state feedback," American Control Conference, 2013.

[10] M.H. Wang, G. Liu, P.T. Zhao, S.H. Yang, "Variable gain state feedback $H_{\infty}$ control for hypersonic vehicle based on LPV," Journal of Astronautics, vol. 34, no. 4, pp. 487-495, 2013.

[11] X. Lan, Y. Wang, L. Liu, "Dynamic decoupling tracking control for the polytopic LPV model of hypersonic vehicle," Science China Information Sciences, vol. 58, no. 9, pp. 1-14, 2015.

[12] H. Shen, K. Liu, B. Chen, Y. Liu, Y. Lu, "Guardian maps based switching tracking control of large envelope hypersonic vehicles," Chinese Control Conference, 2016.

[13] R. A. Garcia-Garcia and M. Arias-Montiel, "A robust control scheme against some parametric uncertainties for the NXT ballbot," Multibody Mechatronic Systems, 249-260, 2015.

[14] H. K. Khalil, L. Praly, "High-gain observers in nonlinear feedback control," International Journal of Robust and Nonlinear Control, Vol. 24, No. 6, pp. 993-1015, 2014.

[15] J. Gallier, "The schur complement and symmetric positive semidefinite (and definite) matrices," Penn Engineering, 2010.

[16] J. Löfberg, "Yalmip: A toolbox for modeling and optimization in Matlab," IEEE International Symposium on Computer Aided Control Systems Design, pp. 284-289, 2004. 